Bulletin of the Section of Logic

Volume 51/1 (2022), pp. 73-89

https://doi.org/10.18778/0138-0680.2021.22

Tomoaki Kawano

\title{
SEQUENT CALCULI FOR ORTHOLOGIC WITH STRICT IMPLICATION
}

\begin{abstract}
In this study, new sequent calculi for a minimal quantum logic (MQL) are discussed that involve an implication. The sequent calculus GO for MQL was established by Nishimura, and it is complete with respect to ortho-models (O-models). As GO does not contain implications, this study adopts the strict implication and constructs two new sequent calculi $\mathbf{G O I}_{1}$ and $\mathbf{G O I}_{2}$ as the expansions of $\mathbf{G O}$. Both $\mathbf{G O I}_{1}$ and $\mathbf{G O I}_{2}$ are complete with respect to the O-models. In this study, the completeness and decidability theorems for these new systems are proven. Furthermore, some details pertaining to new rules and the strict implication are discussed.
\end{abstract}

Keywords: Quantum logic, sequent calculus, completeness theorem, implication, orthologic.

\section{Introduction}

Quantum logic (QL) has been introduced in order to manage strange propositions of quantum physics, such as uncertainty principle. Many structures have been studied to represent and analyze such propositions. In particular, Orthomodular lattices describe the propositional spaces of quantum physics and have been studied as the main structure of QL in the work by Birkhoff and Von Neumann [3]. An orthomodular lattice is based on closed subspaces of a Hilbert space, which is a state space of particles in quantum physics. Instead of these lattices, the Kripke model of QL, the orthomodular model (OM-model), can be used, which also describes a state space

Presented by: Andrzej Indrzejczak

Received: November 23, 2020

Published online: November 9, 2021

(C) Copyright by Author(s), Łódź 2022

(C) Copyright for this edition by Uniwersytet Łódzki, Łódź 2022 
of quantum particles [12]. Ortholattices, which are conceptually simpler than orthomodular lattices, have also been studied. The logic based on ortholattices is a minimal $Q L$ (MQL) or orthologic. Moreover, the Kripke model for MQL, i.e., ortho-model (O-model), also exists [12].

As it is usually studied, QL does not contain logical implications and includes only negations, conjunctions, and disjunctions. Several implications in QL have been suggested; however, they all have difficulties for varying reasons $[12,14]$. Therefore, the deduction systems, such as the Hilbert style axiomatization or sequent calculi that include implications, are not well developed. This problem also holds for MQL. In MQL, the number of appropriate implications is even smaller than that in QL. Therefore, as a part of research to address these problems, this study constructs two new sequent calculi for MQL that include rules for specific implication and provides the completeness theorems with respect to O-models.

When the implications are added to QL or MQL, some problems are encountered. In classical logic, the implication $A \rightarrow B$ and $\neg A \vee B$ can be identified. However, in $\mathbf{Q L}$, if $\neg A \vee B$ is adopted as an implication, critical properties for the implication, such as modus ponens, do not hold. Therefore, in QL, many other implications have been considered. Among them, polynomial implications that can be defined in terms of connectives $\neg, \wedge$ and $\vee$, have been predominantly studied. The polynomial implication Sasaki arrow $\neg A \vee(A \wedge B)$ has attracted the most attention in QL. In addition to the Sasaki arrow, the contrapositive Sasaki arrow $\neg(A \vee B) \vee B$, the relevance arrow $(A \wedge B) \vee(\neg A \wedge B) \vee(\neg A \wedge \neg B)$, and two other arrows have been explored $[12,13,14]$. These implications are the only polynomial implications that have suitable properties in terms of the orthomodular lattice and have been studied from both physical and mathematical standpoints [21].

These implications have been investigated in many ways because of their strangeness. The meaning and properties of these implications in quantum physics are associated with the notion of projections [12, 22]. For example, the Sasaki arrow $\neg A \vee(A \wedge B)$ can be translated as "after a measurement of $A$, if the state is projected to a state which $A$ is true, then $B$ is true." By utilizing this property and embedding the projection relationship in the model, various properties of the Hilbert space can be analyzed using the Kripke model [22]. Recently, these implications have been used in the context of quantum set theory, achieving results in the analysis of observed values in quantum mechanics [29]. The algebraic features of these impli- 
cations have been widely studied in the case of orthomodular lattices and ortholattices $[1,6,4,8,17]$. These studies focus on the logical aspects of orthomodular lattices using implications. Furthermore, concepts regarding orthomodular lattices, such as semilattices, have been analyzed, where implications occupy a principal position [10, 9, 11]. Among them, implication algebras have been discussed as implication studies that exclude other logical operators $[1,7,13,15,16]$. In this field, the properties of orthomodular lattices have been elucidated by analyzing algebraic axioms and conditions for implications. This algebraic research is a purely mathematical study rather than a research related to quantum physics. Few studies on QL have employed binary relational models compared with the number of studied on such algebraic studies. Models using binary relations can express the dynamic relations of quantum physics, and some dynamic concepts are closely related to implications. Therefore, research using the Kripke model, such as that proposed in this study, should be conducted.

However, in ortholattices, polynomial implications do not satisfy modus ponens. In this study, the notion of strict implication proposed in the literature [12] is adopted for MQL, as the strict implication exhibits good mathematical properties, particularly in the Kripke models, and has physically significant meanings. In an ortholattice $L$, strict implication is defined with some restrictions as follows [12]:

$$
a \rightarrow b=\bigsqcup\left\{c \in L \mid c \neq 0 \wedge \forall d\left(\left(d \neq 0 \wedge c \not \leq d^{\prime} \wedge d \leq a\right) \Rightarrow d \leq b\right)\right\}
$$

where $\leq$ is the order in $L, \sqcup$ is the join, and 0 is the least element. Although this definition seems complicated at the first glance, the definition in the Kripke model corresponding to this definition is clear. This is one reason for adopting the Kripke model in the present study. Intuitively, from a quantum physics viewpoint, the strict implication $A \rightarrow B$ can be translated as "after the measurement of any physical quantity, if $A$ is true, then $B$ is true."

Some advantages of the strict implication should be noticed.

- In ortholattices, the Sasaki arrow does not satisfy modus ponens. However, the strict implication satisfies modus ponens in both lattices. Therefore, when MQL is considered, the strict implication is more suitable than the Sasaki arrow. 
- All material implications are abbreviations of formulas constructed using conjunctions, disjunctions, and negations. However, the strict implication cannot be (finitely) constructed by means of these symbols [12]. Therefore, when the strict implication is added to MQL, the descriptive ability of the logic increases.

- The definition of the strict implication in O-models is similar to that of the implication in intuitionistic logic. The deduction rules of the strict implication are similar to those in the sequent calculus LBP for the basic propositional logic (BPL) [20, 31]. Therefore, we can analyze the relationship between $\mathbf{Q L}$ and other logics using this implication.

Although a sequent calculus for MQL with the strict implication exists, a sequent is a labeled type sequent [23]. From the logic viewpoint, it is important to construct and discuss a simple type of sequent calculus for logic. Furthermore, some deduction systems for QL or MQL that involve implications are studied; however, they are either not sequent calculi or the implication used in these systems is not a strict implication [5, 28]. Sequent calculi GO [25] and GMQL [26, 27] have been studied as foundational sequent calculi for MQL which only includes $\neg, \wedge$ and $\vee$. The present study adopts GO for technical reasons, which is presented in Section 6 . The rules for the strict implication are added to GO, and new calculi $\mathbf{G O I}_{1}$ and $\mathbf{G O I}_{2}$ are constructed. This study proves the completeness theorem for these new systems.

Some formulas valid with general implications in other logics are invalid with the strict implication in O-models. For example, $p \rightarrow(q \rightarrow p)$ is invalid. Therefore, general rules for implications, for example, such as those for the implication in classical logic, cannot be used. As mentioned earlier, this study uses a modified version of the rule for the implication of $\mathbf{L B P}$ reported in the literature [20]. The implication of BPL also does not satisfy some ordinary natures of implication. The semantics of this implication in a Kripke model is the same as that of the strict implication. In other words, $x \models A \rightarrow B$ is regarded as "for all $y$, such that $x R y$, if $y \models A$, then $y \models B$."

In Sections 2 and 3, some basics and the sequent calculus of MQL are presented. In Sections 4 and 5 , the new sequent calculi $\mathbf{G O I}_{1}$ and $\mathbf{G O I}_{2}$ are constructed and some related theorems are proven. The deduction 
ability of $\mathbf{G O I}_{1}$ and $\mathbf{G O I}_{2}$ is intrinsically the same; however, the rules for the strict implication are different and each has pros and cons. In Section 6, some details regarding the strict implication and rules are discussed.

\section{Basics}

This study uses language that has a denumerable infinite set of propositional variables, the propositional constant $\perp$, the unary connective $\neg$, and binary connectives $\wedge$ and $\rightarrow$. Formulas are constructed in the usual way. We denote propositional variables by $p, q, \ldots$, formulas by $A, B, C, \ldots$, and finite sets of formulas by $\Gamma, \Delta, \Sigma, \Pi, \ldots$. We use $A \vee B$ as the abbreviation of $\neg(\neg A \wedge \neg B)$.

An $O$-frame is a pair $(W, \perp)$, where $W$ is a nonempty set, and $\perp$ is an irreflexive and symmetric binary relation on $W$. For traditional reasons, we use the symbol $\perp$ in two ways; one as a relation, the other as a formula. The relation symbol $\perp$ came from the orthogonal relation in the Hilbert space, and the formula symbol $\perp$ denotes the bottom. They can be distinguished by the context.

We write $x \not \perp y$ if not $x \perp y$. We write $x \perp X$ if, for all $y \in X, x \perp y$, where $x \in W$ and $X \subseteq W$. Given $X \subseteq W$, we define the set $X^{\perp}=$ $\{x \in W \mid x \perp X\}$. We say that $X$ is $\perp$-closed if $X^{\perp \perp}=X$.

An $O$-model is a triple $(W, \perp, V)$, where $(W, \perp)$ is an O-frame and $V$ is a function assigning each propositional variable $p$ to a $\perp$-closed subset of $W$.

We define the set $\|A\|$ by induction on the composition of $A$ as follows.

$$
\begin{aligned}
& \|p\|=V(p) \\
& \|A \wedge B\|=\|A\| \cap\|B\| \\
& \|\neg A\|=\|A\|^{\perp} \\
& \|A \rightarrow B\|=\{x \in W \mid \text { for all } y \in W, \text { if } x \not \perp y \text { and } y \in\|A\|, y \in\|B\|\} \\
& \|\perp\|=\emptyset
\end{aligned}
$$

$A$ is true at $x$ if $x \in\|A\|$ and write $x \models A$. It is easy to evaluate that $\|\neg A\|=\|A \rightarrow \perp\|$ is fulfilled in this definition. Therefore, we regard $\neg A$ as the abbreviation of $A \rightarrow \perp$. 
Lemma 2.1. For all $\|A\|,\|A\|$ is $\perp$-closed.

Proof: In the cases of $\|p\|,\|A \wedge B\|$ and $\|\neg A\|$, see [25]. For all $x \in \| A \rightarrow$ $B \|, x \perp\{y \in W \mid y \models A$ and $y \not \models B\}$. Then, $\{y \in W \mid y \models A$ and $y \not \models B\} \in$ $\|A \rightarrow B\|^{\perp}$. Therefore, if $z \in\|A \rightarrow B\|^{\perp \perp}$ then $z \perp\{y \in W \mid y \models A$ and $y \not \models B\}$. It means $z \in\|A \rightarrow B\|$. That is, there is no point $z$ which satisfies $z \notin\|A \rightarrow B\|$ and $z \in\|A \rightarrow B\|^{\perp \perp}$. Therefore, $\|A \rightarrow B\|$ is $\perp$-closed.

\section{Sequent calculus GO}

GO is defined below [25].

Axiom:

$$
A \Rightarrow A
$$

Rules:

$$
\begin{aligned}
& \frac{\Gamma \Rightarrow \Delta, A \quad A, \Pi \Rightarrow \Sigma}{\Gamma, \Pi \Rightarrow \Delta, \Sigma} \text { (cut) } \frac{\Gamma \Rightarrow \Delta}{\Pi, \Gamma \Rightarrow \Delta, \Sigma} \text { (weakening) } \\
& \frac{A, \Gamma \Rightarrow \Delta}{A \wedge B, \Gamma \Rightarrow \Delta}(\wedge \mathrm{L}) \quad \frac{B, \Gamma \Rightarrow \Delta}{A \wedge B, \Gamma \Rightarrow \Delta}(\wedge \mathrm{L}) \quad \frac{\Gamma \Rightarrow \Delta, A \quad \Gamma \Rightarrow \Delta, B}{\Gamma \Rightarrow \Delta, A \wedge B}(\wedge \mathrm{R}) \\
& \frac{\Gamma \Rightarrow \Delta, A}{\neg A, \Gamma \Rightarrow \Delta}(\neg \mathrm{L}) \quad \frac{A \Rightarrow \Delta}{\neg \Delta \Rightarrow \neg A}(\neg \mathrm{R}) \\
& \frac{A, \Gamma \Rightarrow \Delta}{\neg \neg A, \Gamma \Rightarrow \Delta}(\neg \neg \mathrm{L}) \quad \frac{\Gamma \Rightarrow \Delta, A}{\Gamma \Rightarrow \Delta, \neg \neg A}(\neg \neg \mathrm{R})
\end{aligned}
$$

In [25], $\Gamma, \Delta, \Pi$ and $\Sigma$ are defined as probable infinite sets. We restrict these to finite sets because infinite sets are not essential here.

Consider an O-model $(W, \perp, V)$. Sequent $\Gamma \Rightarrow \Delta$ is false at $x \in W$ if for all formulas $A \in \Gamma, x \models A$, and, for all formulas $B \in \Delta, x \not \models B$. If $\Gamma \Rightarrow \Delta$ is not false at $x$, then it is true at $x$. Sequent $\Gamma \Rightarrow \Delta$ is falsifiable if there exists an O-model $(W, \perp, V)$ and $x \in W$, and $\Gamma \Rightarrow \Delta$ is false at $x$. If $\Gamma \Rightarrow \Delta$ is unfalsifiable, we say $\Gamma \Rightarrow \Delta$ is valid.

THEOREM 3.1. The soundness and completeness theorem for GO. $\Gamma \Rightarrow \Delta$ is provable in $\mathbf{G O}$ if, and only if, (iff) $\Gamma \Rightarrow \Delta$ is valid.

Proof: See [25]. 


\section{Sequent calculus GOI $_{1}$}

In this section, a sequent calculus including the strict implication is established. The sequent calculus $\mathbf{G O I}_{1}$ is defined as an expansion of $\mathbf{G O}$. The rule $(\rightarrow \mathrm{R})$ and axiom $\perp \Rightarrow$ are added to GO. The rule $(\rightarrow \mathrm{R})$ is the transformation of the rule $(\rightarrow)$ in [20]. Because this rule $(\rightarrow \mathrm{R})$ is complex, using $\mathbf{G O I}_{2}$ in the next chapter for the main calculus of $\mathbf{M Q L}$ with the strict implication would be better. However, $(\rightarrow \mathrm{R})$ is useful to prove the completeness theorem. Therefore, first the details of $\mathbf{G O I}_{1}$ are shown. The definitions of truth, falsity, and validity of a sequent are identical to that in GO.

$$
\begin{gathered}
\perp \Rightarrow(\perp) \\
\frac{\Gamma_{1}, A \Rightarrow B, \Delta_{1}, \Sigma \quad \Gamma_{2}, A \Rightarrow B, \Delta_{2}, \Sigma \ldots \Gamma_{2^{n}}, A \Rightarrow B, \Delta_{2^{n}}, \Sigma}{C_{1} \rightarrow D_{1}, C_{2} \rightarrow D_{2}, \ldots, C_{n} \rightarrow D_{n}, \Pi \Rightarrow A \rightarrow B, \Lambda} \quad(\rightarrow \mathrm{R})
\end{gathered}
$$

where, $0 \leq n, \Gamma_{i}=\left\{D_{j} \mid j \in \gamma(i)\right\}, \Delta_{i}=\left\{C_{j} \mid j \in \delta(i)\right\},\langle\delta(i), \gamma(i)\rangle$ is the $i$-th element of all partitions of $\{1, \ldots, n\}$. $\Pi$ and $\Lambda$ are formula sets. $\Sigma$ is a set of all formulas of the shape $E \rightarrow F$ such that $E$ is included in the premise of the lower sequent and $F$ is included in the conclusion of the lower sequent or $\perp$. Therefore, $\Sigma=\left\{E \rightarrow F \mid E \in\left\{C_{1} \rightarrow D_{1}, \ldots, C_{n} \rightarrow\right.\right.$ $\left.\left.D_{n}, \Pi\right\}, F \in\{A \rightarrow B, \Lambda, \perp\}\right\}$.

For example, suppose $\Pi=\{I\}, \Lambda=\{J, K\}$, then $(\rightarrow \mathrm{R})$ is as below in the case of $n=0, n=1$, and $n=2$.

$$
\begin{gathered}
\frac{A \Rightarrow B, I \rightarrow(A \rightarrow B), I \rightarrow J, I \rightarrow K, I \rightarrow \perp}{I \Rightarrow A \rightarrow B, J, K} \\
\frac{A \Rightarrow B, C_{1}, \Sigma \quad D_{1}, A \Rightarrow B, \Sigma}{C_{1} \rightarrow D_{1}, I \Rightarrow A \rightarrow B, J, K}
\end{gathered}
$$

where $\Sigma$ is $\left\{\left(C_{1} \rightarrow D_{1}\right) \rightarrow(A \rightarrow B),\left(C_{1} \rightarrow D_{1}\right) \rightarrow J,\left(C_{1} \rightarrow D_{1}\right) \rightarrow\right.$ $\left.K,\left(C_{1} \rightarrow D_{1}\right) \rightarrow \perp, I \rightarrow(A \rightarrow B), I \rightarrow J, I \rightarrow K, I \rightarrow \perp\right\}$.

$$
\frac{A \Rightarrow B, C_{1}, C_{2}, \Sigma \quad D_{1}, A \Rightarrow B, C_{2}, \Sigma \quad D_{2}, A \Rightarrow B, C_{1}, \Sigma \quad D_{1}, D_{2}, A \Rightarrow B, \Sigma}{C_{1} \rightarrow D_{1}, C_{2} \rightarrow D_{2}, I \Rightarrow A \rightarrow B, J, K}
$$


where $\Sigma$ is $\left\{\left(C_{1} \rightarrow D_{1}\right) \rightarrow(A \rightarrow B),\left(C_{1} \rightarrow D_{1}\right) \rightarrow J,\left(C_{1} \rightarrow D_{1}\right) \rightarrow\right.$ $K,\left(C_{1} \rightarrow D_{1}\right) \rightarrow \perp,\left(C_{2} \rightarrow D_{2}\right) \rightarrow(A \rightarrow B),\left(C_{2} \rightarrow D_{2}\right) \rightarrow J,\left(C_{2} \rightarrow\right.$ $\left.\left.D_{2}\right) \rightarrow K,\left(C_{2} \rightarrow D_{2}\right) \rightarrow \perp, I \rightarrow(A \rightarrow B), I \rightarrow J, I \rightarrow K, I \rightarrow \perp\right\}$.

In $\mathbf{G O I}_{1}$, the rule $(\rightarrow \mathrm{L})$ is admissible.

$$
\frac{\Gamma_{1}, \Rightarrow \Delta_{1}, A \quad B, \Gamma_{2} \Rightarrow \Delta_{2}}{\Gamma_{1}, \Gamma_{2}, A \rightarrow B \Rightarrow \Delta_{1}, \Delta_{2}}(\rightarrow \mathrm{L})
$$

We will prove this lemma in Section 5 .

ThEOREM 4.1. The soundness theorem for $\mathbf{G O I}_{1}$. If $\Gamma \Rightarrow \Delta$ is provable in $\mathbf{G O I}_{1}, \Gamma \Rightarrow \Delta$ is valid.

Proof: Proven by induction on the construction of a proof. For rules in GO, the proof is the same as the proof in [25]. For $(\rightarrow \mathrm{R})$, we only evaluate $n=2$. The other cases are similar. For contradiction, suppose all premises of the rule are valid, and there exists O-model $(W, \perp, V)$ and $x \in W$, such that the conclusion of the rule is false at $x$. Then, as $A \rightarrow B$ is false at $x$, there exists $y \in W$, satisfying $x \not \perp y, y \models A$ and $y \not \models B$. Because we assume that $y \models A$ and all premises are valid, from the first premise, $B$ or $C_{1}$ or $C_{2}$ or one of the formulas in $\Sigma$ is true at $y$; however, $B$ is false at $y$. Now, suppose $E \rightarrow F \in \Sigma$. We have $x \models E$ and $x \not \models F$ by assumption and the definition of $(\rightarrow \mathrm{R})$. If $y \models E \rightarrow F$, from $y \not \perp x$ and $x \models E, x \models F$, which is a contradiction. Therefore, for all $E \rightarrow F \in \Sigma, y \not \models E \rightarrow F$. Therefore, $C_{1}$ or $C_{2}$ is true at $y$. In the former case, from $x \models C_{1} \rightarrow D_{1}$ and $y \models C_{1}$, $y \models D_{1}$. From the second premise, $B$ or $C_{2}$ or one of the formulas in $\Sigma$ is true at $y$. Similarly, the only possibility is $C_{2}$; therefore, $C_{2}$ is true at $y$. To continue this method to the end of premises, $B$ or $\Sigma$ is the only possibility, which is a contradiction. The latter case and cases of the other possibilities are similar to this method.

To prove the completeness theorem, we define the set $\Omega$ as follows. $\Omega(\Gamma \Rightarrow \Delta)=\{$ All subformulas in $\Gamma \cup \Delta\} \cup\{\neg p \mid p$ appear in some formulas in $\Gamma \cup \Delta\} \cup\{\perp\}$. For example, $\Omega(\neg(p \rightarrow q) \Rightarrow r \wedge q)=$ $\{\perp, p, q, r, \neg p, \neg q, \neg r, p \rightarrow q, r \wedge q, \neg(p \rightarrow q)\}$. For each unprovable sequent $\Gamma \Rightarrow \Delta$, we define a canonical $O$-model $\left(W_{c}, \perp_{c}, V_{c}\right)$ of $\Gamma \Rightarrow \Delta$ as follows.

$W_{c}:\left\{\Gamma_{1} \Rightarrow \Delta_{1} \mid \Gamma_{1} \Rightarrow \Delta_{1}\right.$ is unprovable in $\mathbf{G O I}_{1}$ and $\Gamma_{1} \cup \Delta_{1}=\Omega(\Gamma \Rightarrow$ $\Delta)\}$ 
$\perp_{c}:\left(\Gamma_{1} \Rightarrow \Delta_{1}\right) \perp\left(\Gamma_{2} \Rightarrow \Delta_{2}\right)$ iff for some $A$ and $B$, at least one of (1) (2) is true. (1) $A \rightarrow B \in \Gamma_{1}, A \in \Gamma_{2}$ and $B \in \Delta_{2}$. (2) $A \rightarrow B \in \Gamma_{2}$, $A \in \Gamma_{1}$ and $B \in \Delta_{1}$.

$V_{c}$ : assigns $p$ to the set $\left\{\Gamma_{1} \Rightarrow \Delta_{1} \mid p \notin \Delta_{1}\right\}$.

Lemma 4.2. $\left(W_{c}, \perp_{c}\right)$ is an $O$-frame. $V_{c}(p)$ is $\perp$-closed. Therefore, $\left(W_{c}, \perp_{c}\right.$, $\left.V_{c}\right)$ is an $O$-model.

Proof: If $\left(\Gamma_{1} \Rightarrow \Delta_{1}\right) \perp\left(\Gamma_{1} \Rightarrow \Delta_{1}\right)$, there is $A$ and $B$ that $A \in \Gamma_{1}, A \rightarrow$ $B \in \Gamma_{1}$ and $B \in \Delta_{1}$. But $A, A \rightarrow B \Rightarrow B$ is proven using ( $\left.\rightarrow \mathrm{L}\right)$; therefore, $\Gamma_{1} \Rightarrow \Delta_{1}$ can be proven, which is a contradiction. Therefore, for every $\Gamma_{1} \Rightarrow \Delta_{1} \in W_{C},\left(\Gamma_{1} \Rightarrow \Delta_{1}\right) \not \perp\left(\Gamma_{1} \Rightarrow \Delta_{1}\right)$. Symmetry is obvious from the definition. If $p \notin \Omega(\Gamma \Rightarrow \Delta), V_{c}(p)=W_{c}$. This is clearly $\perp$-closed. If $p \in \Omega(\Gamma \Rightarrow \Delta)$, for every $\Gamma_{1} \Rightarrow \Delta_{1} \in W_{c}, p \in \Gamma_{1}$ or $p \in \Delta_{1}$. Then, $\left(\Gamma_{1} \Rightarrow \Delta_{1}\right) \models p$ iff $p \in \Gamma_{1}$. Therefore, if we can prove the next statement, we can prove this lemma.

For all $\left(\Gamma_{1} \Rightarrow \Delta_{1}\right) \in W_{c}$, if $p \in \Delta_{1}$, there exists $\left(\Gamma_{2} \Rightarrow \Delta_{2}\right) \in W_{C}$, satisfying $\neg p \in \Gamma_{2}$ and $\left(\Gamma_{1} \Rightarrow \Delta_{1}\right) \not \perp\left(\Gamma_{2} \Rightarrow \Delta_{2}\right)$.

For convenience, we prove this statement after the next lemma.

Lemma 4.3. For all canonical $O$-models and all formulas $A \in \Omega, A$ is true at $\left(\Gamma_{1} \Rightarrow \Delta_{1}\right)$ if $A \in \Gamma_{1}$ and $A$ is false at $\left(\Gamma_{1} \Rightarrow \Delta_{1}\right)$ if $A \in \Delta_{1}$.

Proof: Proven by induction on the composition of $A$.

For $A=p$, the proof is obvious from the definition of a canonical Omodel.

For $A=B \wedge C$, the proof is the same as in [25].

For $A=\neg B$, the proof is included in $A=B \rightarrow C$.

For $A=B \rightarrow C$, suppose $B \rightarrow C \in \Gamma_{1}$. Then, for all $\left(\Gamma_{2} \Rightarrow \Delta_{2}\right)$ satisfying $B \in \Gamma_{2}$ and $C \in \Delta_{2},\left(\Gamma_{1} \Rightarrow \Delta_{1}\right) \perp\left(\Gamma_{2} \Rightarrow \Delta_{2}\right)$ by the definition of the canonical O-model. Then, by definition of $\rightarrow$ and induction hypothesis, $B \rightarrow C$ is true at $\left(\Gamma_{1} \Rightarrow \Delta_{1}\right)$.

Suppose $B \rightarrow C \in \Delta_{1}$. Because $\Gamma_{1} \Rightarrow \Delta_{1}$ cannot be proven, when we regard this sequent as the lower sequent of the rule $(\rightarrow \mathrm{R})$, an unprovable sequent $\Gamma_{2}, B \Rightarrow C, \Delta_{2}$ exists, which is of the shape of a sequent in the upper sequent of $(\rightarrow \mathrm{R})$. Then, $\Gamma_{2}$ and $\Delta_{2}$ distribute all formulas of the 
shape of $E \rightarrow F$ in $\Gamma_{1}$, regarded as a $C_{i} \rightarrow D_{i}$. If there are formulas in $\Gamma_{2}, B \Rightarrow C, \Delta_{2}$ that are excluded in $\Omega(\Gamma \Rightarrow \Delta)$, we delete them from $\Gamma_{2}, B \Rightarrow C, \Delta_{2}$ and make a new sequent $\Gamma_{3}, B \Rightarrow C, \Delta_{3}$. Then, $\Gamma_{3} \cup$ $\{B, C\} \cup \Delta_{3} \subseteq \Omega(\Gamma \Rightarrow \Delta)$ and this sequent is still unprovable. This sequent can be expanded to the sequent $\Gamma_{4} \Rightarrow \Delta_{4} \in W_{c}$ because for all formulas $G$, at least one $\Gamma_{3}, B \Rightarrow C, \Delta_{3}, G$ or $G, \Gamma_{3}, B \Rightarrow C, \Delta_{3}$ is unprovable because of the rule (cut) and because $\Gamma_{3}, B \Rightarrow C, \Delta_{3}$ is unprovable. Furthermore, $\left(\Gamma_{1} \Rightarrow \Delta_{1}\right) \not \perp\left(\Gamma_{4} \Rightarrow \Delta_{4}\right)$ is satisfied because we delete all probability of holding the relation $\perp$ when we construct $\Gamma_{2}, B \Rightarrow C, \Delta_{2}$. Therefore, by the definition of $\rightarrow$ and induction hypothesis, $B \rightarrow C$ is false at $\Gamma_{1} \Rightarrow \Delta_{1}$.

Now we can prove the statement in Lemma 4.2 using the method of the proof of Lemma 4.3. If $\Gamma_{1} \Rightarrow \Delta_{1}, p\left(\in W_{c}\right)$ is unprovable, $\Gamma_{1} \Rightarrow$ $\Delta_{1}, p, \neg \neg p$ is also unprovable. We regard $(p \rightarrow \perp) \rightarrow \perp$ as $B \rightarrow C$ in Lemma 4.3. The same argument for $B \rightarrow C \in \Delta_{1}$ in Lemma 4.3 can be applied. That is, we can find $\left(\Gamma_{4} \Rightarrow \Delta_{4}\right) \in W_{c}$, satisfying $\neg p \in \Gamma_{4}$, $\perp \in \Delta_{4}$, and $\left(\Gamma_{1} \Rightarrow \Delta_{1}, p\right) \not \perp\left(\Gamma_{4} \Rightarrow \Delta_{4}\right)$. If $\neg \neg p$ is included in $\Omega(\Gamma \Rightarrow \Delta)$, $\Gamma_{1} \Rightarrow \Delta_{1}, p, \neg \neg p$ is the same as $\Gamma_{1} \Rightarrow \Delta_{1}, p$ and is included in $W_{c}$. If $\neg \neg p$ is excluded in $\Omega(\Gamma \Rightarrow \Delta)$, sequent $\Gamma_{4} \Rightarrow \Delta_{4}\left(\neg p \in \Gamma_{4}\right)$, constructed from $\Gamma_{1} \Rightarrow \Delta_{1}, p, \neg \neg p$ is included in $W_{c}$, even if $\Gamma_{1} \Rightarrow \Delta_{1}, p, \neg \neg p$ is excluded in $W_{c}$. That is, when we make $\Gamma_{3}, \neg p \Rightarrow \perp, \Delta_{3}$ from $\Gamma_{1} \Rightarrow \Delta_{1}, p, \neg \neg p$, we eliminate all formulas that are excluded in $\Omega(\Gamma \Rightarrow \Delta)$. Furthermore, it satisfies $\left(\Gamma_{1} \Rightarrow \Delta_{1}, p\right) \not \perp\left(\Gamma_{4} \Rightarrow \Delta_{4}\right)$ because $\Gamma_{1} \Rightarrow \Delta_{1}, p$ is a part of $\Gamma_{1} \Rightarrow \Delta_{1}, p, \neg \neg p$.

THEOREM 4.4. The completeness theorem for $\mathbf{G O I}_{1}$. If $\Gamma \Rightarrow \Delta$ is valid, $\Gamma \Rightarrow \Delta$ is provable in $\mathbf{G O I}_{1}$.

Proof: Suppose $\Gamma \Rightarrow \Delta$ is unprovable. We can make a canonical Omodel of $\Gamma \Rightarrow \Delta$. Because (cut) is included in $\mathbf{G O I}_{1}$, there exists $\left(\Gamma^{\prime} \Rightarrow\right.$ $\left.\Delta^{\prime}\right) \in W_{c}$, an expansion of $\Gamma \Rightarrow \Delta$. By Lemma $4.3, \Gamma \Rightarrow \Delta$ is false at $\left(\Gamma^{\prime} \Rightarrow \Delta^{\prime}\right)$.

\section{Sequent calculus $\mathrm{GOI}_{2}$}

We define the sequent calculus $\mathbf{G O I}_{2}$ as an expansion of $\mathbf{G O}$. We add the axioms $(\rightarrow \perp)$ and $(\perp)$ and the rule $(\rightarrow \mathrm{R})^{\prime}$ to GO. The rule $(\rightarrow \mathrm{R})^{\prime}$ ' is similar to the rule $(\rightarrow)$ in [20], but there are no contexts in this rule. 


$$
\begin{gathered}
\perp \Rightarrow \quad(\perp) \\
A \Rightarrow(A \rightarrow B) \rightarrow \perp, B \quad(\rightarrow \perp) \\
\frac{\Gamma_{1}, A \Rightarrow B, \Delta_{1} \quad \Gamma_{2}, A \Rightarrow B, \Delta_{2} \quad \ldots \quad \Gamma_{2^{n}}, A \Rightarrow B, \Delta_{2^{n}}}{C_{1} \rightarrow D_{1}, C_{2} \rightarrow D_{2}, \ldots, C_{n} \rightarrow D_{n} \Rightarrow A \rightarrow B}(\rightarrow \mathrm{R})^{\prime}
\end{gathered}
$$

where $0 \leq n, \Gamma_{i}=\left\{D_{j} \mid j \in \gamma(i)\right\}, \Delta_{i}=\left\{C_{j} \mid j \in \delta(i)\right\},\langle\delta(i), \gamma(i)\rangle$ is the $i$-th element of all partitions of $\{1, \ldots, n\}$.

The rule $(\rightarrow \mathrm{R})^{\prime}$ ' is a natural expansion of the rule $(\neg R)$ in GO. That is, if all $D_{j}$ and $B$ in $(\rightarrow \mathrm{R})^{\prime}$ are $\perp$, it is the same as $(\neg R)$ in $\mathbf{G O}$ because of $A \rightarrow \perp \equiv \neg A$.

TheOREM 5.1. The soundness and completeness theorem for $\mathbf{G O I}_{2} . \Gamma \Rightarrow$ $\Delta$ is provable in $\mathbf{G O I}_{2}$ iff $\Gamma \Rightarrow \Delta$ is valid.

Proof: We can prove that all rules of $\mathbf{G O I}_{1}$ are derivable in $\mathbf{G O I}_{2}$, and vice versa. The proof of $(\rightarrow \perp)$ in $\mathbf{G O I}_{1}$ and $(\rightarrow \mathrm{R})$ in $\mathbf{G O I}_{2}$ is explained below. The other cases are obvious.

$$
\frac{A \rightarrow B \Rightarrow A \rightarrow B}{\frac{A \rightarrow B \Rightarrow \perp, A \rightarrow B, A \rightarrow((A \rightarrow B) \rightarrow \perp), A \rightarrow \perp}{A \Rightarrow(A \rightarrow B) \rightarrow \perp, B}}(\rightarrow \mathrm{R})
$$

Suppose all sequents of upper sequents in $(\rightarrow \mathrm{R})$ are provable. For example, suppose $n=2$. Then,

$$
\begin{aligned}
& A \Rightarrow B, C_{1}, C_{2}, \Sigma \\
& D_{1}, A \Rightarrow B, C_{2}, \Sigma \\
& D_{2}, A \Rightarrow B, C_{1}, \Sigma \\
& D_{1}, D_{2}, A \Rightarrow B, \Sigma
\end{aligned}
$$

are all provable. Now we regard all formulas in $\Sigma$ as a $C_{i}(n<i)$. For example, if $\Sigma$ has three elements, we regard $\Sigma$ as $\left\{C_{3}, C_{4}, C_{5}\right\}$. Furthermore, we define all $D_{i}(n<i)$ as $D_{i}=\perp$. Then,

$$
\begin{aligned}
& A \Rightarrow B, C_{1}, C_{2}, C_{3}, C_{4}, C_{5} \\
& D_{1}, A \Rightarrow B, C_{2}, C_{3}, C_{4}, C_{5} \\
& \ldots \\
& D_{1}, D_{2}, D_{3}, D_{4}, D_{5}, A \Rightarrow B
\end{aligned}
$$

are all provable because, if all formulas in $\Sigma=\left\{C_{3}, C_{4}, C_{5}\right\}$ are on the right-hand side, it is obvious from the assumption. If one of a $\left\{D_{3}, D_{4}, D_{5}\right\}$ 
is on the left-hand side and because all are $\perp$, this sequent is provable. We can use all these sequents and use $(\rightarrow \mathrm{R})^{\prime}$. Then, because $E \Rightarrow(E \rightarrow F) \rightarrow$ $\perp, F$ is provable using $(\rightarrow \perp)$, use (cut), and prove the lower sequent of $(\rightarrow \mathrm{R})$.

TheOrem 5.2. In $\mathbf{G O I}_{1}$ and $\mathbf{G O I}_{2}$, the rule $(\rightarrow L)$ is admissible.

Proof: By using (cut) and because $A, A \rightarrow B \Rightarrow B$ is provable in these systems,

$$
\frac{\frac{A \rightarrow B \Rightarrow A \rightarrow B}{A \rightarrow B, \Rightarrow((A \rightarrow B) \rightarrow \perp) \rightarrow \perp} \quad \frac{A \Rightarrow(A \rightarrow B) \rightarrow \perp, B}{A,((A \rightarrow B) \rightarrow \perp) \rightarrow \perp \Rightarrow B}}{A, A \rightarrow B \Rightarrow B}
$$

Because the canonical model $\left(W_{c}, \perp_{c}, V_{c}\right)$ finite, we can prove the following theorem using the usual method as in GO.

THEOREM 5.3. $\mathbf{G O I}_{1}$ and $\mathbf{G O I}_{2}$ are decidable. That is, an effective procedure determines whether a sequent $\Gamma \Rightarrow \Delta$ is provable in $\mathbf{G O I}_{1}$ and $\mathbf{G O I}_{2}$.

Proof: From the construction method of the canonical model $\left(W_{c}, \perp_{c}, V_{c}\right)$, built from a sequent $\Gamma \Rightarrow \Delta$, we obtain a finite model for any $\Gamma \Rightarrow \Delta$, and the model's complexity can be bounded by the complexity of formulas and the number of propositional letters in $\Gamma$ and $\Delta$. Therefore, by evaluating all finite models up to the bound, whether sequent $\Gamma \Rightarrow \Delta$ is valid can be determined. From the soundness and completeness theorem, this method can determine whether $\Gamma \Rightarrow \Delta$ is provable in $\mathbf{G O I}_{1}$ and $\mathbf{G O I}_{2}$.

\section{Conclusion and remarks}

This study introduced two sequent calculi for MQL that involve the strict implication. The rule for the implication in $\mathbf{G O I}_{1}$ is complicated. On the contrary, the rule for the implication in $\mathbf{G O I}_{2}$ is less complicated and it is a natural expansion of the rule $(\neg R)$. However, the axiom $(\rightarrow \perp)$ must be included in $\mathbf{G O I}_{2}$. In both the calculi, the cut-elimination theorem does not hold. In actuality, $p, q \Rightarrow \neg(r \wedge \neg(p \wedge q))$ cannot be proven without (cut), as in GO [25]. In other words, based on the rules for 
$\mathbf{G O I}_{1}$ and $\mathbf{G O I}_{2}$, in the proof of $p, q \Rightarrow \neg(r \wedge \neg(p \wedge q))$, we can only use (weakening), (cut), or $(\rightarrow \mathrm{R})$ to deduce $p, q \Rightarrow \neg(r \wedge \neg(p \wedge q))$. However, it is easy to confirm that (weakening) does not work. Additionally, $r \wedge \neg(p \wedge q) \Rightarrow \perp, \neg p, \neg q, p \rightarrow \neg(r \wedge \neg(p \wedge q)), q \rightarrow \neg(r \wedge \neg(p \wedge q))$ can be checked for invalidity. Moreover, it is challenging to construct a sequent calculus for QL and MQL that satisfies the cut-elimination theorem using an ordinary method. The situation is similar to that in the modal logic S5. Both S5 and QL exhibit a symmetric frame. If an attempt is made to construct a canonical model of the $\mathbf{S 5}$-frame in a stepwise manner, the procedure cannot be stopped because of the symmetry. An effective tool for addressing this problem is an extension of the sequent calculus. Various extensions of sequence calculus for S5 have been constructed and analyzed $[2,18,19,24,30]$. As one of them, labeled sequent calculi or tree sequent calculi have been studied. A labeled sequent calculus for MQL with the strict implication has been established and is cut-free [23]. It is still an open question whether a normal sequent calculus for MQL that satisfies the cut-elimination theorem exists.

In BPL, the law of modus ponens does not hold [20]. Modus ponens $A, A \rightarrow B \Rightarrow B$ represents the reflexive condition of relations in frames which is not the nature of frames of BPL. Therefore, the rule $(\rightarrow \mathrm{L})$ is not sound in LBP. $(\rightarrow \mathrm{L})$ cannot be constructed if only $(\rightarrow \mathrm{R})$ ' exists for the implication. In $\mathbf{G O I}_{1}$ and $\mathbf{G O I}_{2}$, because other rules or axioms for the implication are included, $(\rightarrow \mathrm{L})$ can be constructed.

Another sequent calculus for MQL called GMQL [26, 27] is also complete with respect to O-models and exclude implications, similar to GO. In GO, based on the definition of the truth of a sequent, $\Gamma \Rightarrow \Delta, A, B$ cannot be regarded as $\Gamma \Rightarrow \Delta, A \vee B$ because commas on the right side of the sequent indicate a union of sets. $\|A\| \cup\|B\|$ and $\|A \vee B\|$ are different sets in O-models, and $\|A\| \cup\|B\|$ is not always $\perp$-closed. For example, $\Rightarrow A, \neg A$ cannot be proven in GO; however, $\Rightarrow A \vee \neg A(=\Rightarrow \neg(\neg A \wedge \neg \neg A))$ can be proven. In GMQL, $\Gamma \Rightarrow \Delta, A, B$ represent $\Gamma \Rightarrow \Delta, A \vee B$. Because the rules in GMQL are close to the notion of a lattice, the rules for $\wedge$ and $\vee$ in GMQL are symmetric because $\wedge$ and $\vee$ are symmetric in ortholattices. In the case of GO, that excludes an implication, this notion of a union of sets is inessential because of the following theorem [20]. 
THEOREM 6.1. If $\Gamma \Rightarrow \Delta$ is provable in GO and $\Delta$ is nonempty, then there exists $A \in \Delta$ such that $\Gamma \Rightarrow A$ is provable and all sequents in that proof have at most one formula on the right side.

When considering the rules for implications, GMQL is unsuitable because in the rules for strict implication, the notion of a union of sets on the right side of a sequent is used rather than $V$. In the case of $\mathbf{G O I}_{1}$ and $\mathbf{G O I}_{2}$, the notion of a union of sets is essential and Theorem 6.1 does not hold in these calculi. This finding can be confirmed by considering the axiom $(\rightarrow \perp)$ and the completeness theorem. In other words, both $A \Rightarrow(A \rightarrow B) \rightarrow \perp$ and $A \Rightarrow B$ are invalid.

In a sense, the axiom $(\rightarrow \perp)$ represents the symmetry of the relation in frames. If $\mathbf{G O I}_{2}$ includes only $(\rightarrow \mathrm{R})^{\prime}$ ' for the strict implication, the symmetry cannot be handled because $(\rightarrow \mathrm{R})^{\prime}$ is a part of the sequent calculus reported in the literature [20] which is sound and complete with respect to the frames that do not need to be symmetrical. Assume that in an Omodel $(W, \perp, V), x \models A$ and $x \not \models B$, then for all $y \in W$ such that $x \not \perp y$, $y \not \vDash A \rightarrow B$ attributed is the symmetry of $\not \subset$. If $B=\perp$, then the axiom $(\rightarrow \perp)$ is $A \Rightarrow \neg \neg A$. When the translation in the literature [12] which translate a formula of QL to a formula of modal logic is applied, this sequent corresponds to $A \Rightarrow \square \diamond A$, representing the symmetry in the modal logic.

In the rule $(\rightarrow)$ in $\mathbf{L B P}$, in every left side of the sequent, contexts can be used. Therefore, $p \rightarrow(q \rightarrow p)$ can be proven in a sequent calculus for LBP using $n=0$ of $(\rightarrow)$, which cannot be proven in $\mathbf{G O I}_{1}$.

$$
\begin{gathered}
\frac{\Gamma, A \Rightarrow B}{\Gamma \Rightarrow A \rightarrow B}(n=0 \text { of }(\rightarrow) \text { in LBP }) \\
\frac{\frac{p \Rightarrow p}{q, p \Rightarrow p}}{p \Rightarrow q \rightarrow p} \\
\Rightarrow p \rightarrow(q \rightarrow p)
\end{gathered}
$$

Acknowledgements. I would like to thank R. Goré, R. Kashima and the anonymous reviewers for helpful comments on earlier version of this paper.

This work was supported by JSPS KAKENHI Grant Number JP20K19740. 


\section{References}

[1] J. C. Abbott, Orthoimplication Algebras, Studia Logica, vol. 35(2) (1976), pp. 173-177, DOI: https://doi.org/10.1007/BF02120879.

[2] K. Bednarska, A. Indrzejczak, Hypersequent Calculi for S5 - The Methods of Cut-elimination, Logic and Logical Philosophy, vol. 24(3) (2015), pp. 277-311, DOI: https://doi.org/10.12775/LLP.2015.018.

[3] G. Birkhoff, J. V. Neumann, The Logic of Quantum Mechanics, Annals of Mathematics, vol. 37(4) (1936), pp. 823-843, DOI: https://doi.org/10. $2307 / 1968621$.

[4] I. Chajda, The axioms for implication in orthologic, Czechoslovak Mathematical Journal, vol. 58(1) (2008), pp. 735-744, DOI: https://doi.org/ 10.1007/s10587-008-0002-2.

[5] I. Chajda, J. Cirulis, An Implicational Logic for Orthomodular Lattices, Acta Scientiarum Mathematicarum, vol. 82(34) (2016), pp. 383-394, DOI: https://doi.org/10.14232/actasm-015-813-6.

[6] I. Chajda, R. Halaš, An Implication in Orthologic, International Journal of Theoretical Physics, vol. 44(7) (2005), pp. 735-744, DOI: https://doi. org/10.1007/s10773-005-7051-1.

[7] I. Chajda, R. Halaš, H. Länger, Orthomodular Implication Algebras, International Journal of Theoretical Physics, vol. 40(11) (2001), pp. 18751884, DOI: https://doi.org/10.1023/A:1011933018776.

[8] I. Chajda, H. Länger, Orthomodular Posets Can Be Organized as Conditionally Residuated Structures, Acta Universitatis Palackianae Olomucensis. Facultas Rerum Naturalium. Mathematica, vol. 53(2) (2014), pp. 29-33, URL: https://kma.upol.cz/data/xinha/ULOZISTE/ActaMath/ 2014/53-2-02.pdf.

[9] I. Chajda, H. Länger, Orthomodular lattices can be converted into leftresiduated l-groupoids, Miskolc Mathematical Notes, vol. 18(2) (2017), pp. 685-689, DOI: https://doi.org/10.18514/mmn.2017.1730.

[10] I. Chajda, H. Länger, Residuation in orthomodular lattices, Topological Algebra and its Applications, vol. 5(1) (2017), pp. 1-5, DOI: https: //doi.org/10.1515/taa-2017-0001.

[11] I. Chajda, H. Länger, How to introduce the connective implication in orthomodular posets, Asian-European Journal of Mathematics, vol. 14(4) (2021), p. 2150066, DOI: https://doi.org/10.1142/S1793557121500662. 
[12] M. L. D. Chiara, R. Giuntini, Quantum Logics, [in:] D. M. Gabbay, F. Guenthner (eds.), Handbook of Philosophical Logic, Springer Netherlands, Dordrecht (2002), pp. 129-228, DOI: https://doi.org/10.1007/978-94-0170460-1_2.

[13] P. D. Finch, Quantum logic as an implication algebra, Bulletin of the Australian Mathematical Society, vol. 2(1) (1970), pp. 101-106, DOI: https://doi.org/10.1017/S0004972700041642.

[14] G. M. Hardegree, Material Implication in Orthomodular (and Boolean) Lattices, Notre Dame Journal of Formal Logic, vol. 22(2) (1981), pp. 163182, DOI: https://doi.org/10.1305/ndjfl/1093883401.

[15] G. M. Hardegree, Quasi-implication algebras, Part I: Elementary theory, Algebra Universalis, vol. 12(1) (1981), pp. 30-47, DOI: https://doi.org/ 10.1007/BF02483861.

[16] G. M. Hardegree, Quasi-implication algebras, Part II: Structure theory, Algebra Universalis, vol. 12(1) (1981), pp. 48-65, DOI: https: //doi.org/10.1007/BF02483862.

[17] L. Herman, E. L. Marsden, R. Piziak, Implication Connectives in Orthomodular Lattices, Notre Dame Journal of Formal Logic, vol. 16(3) (1975), pp. 305-328, DOI: https://doi.org/10.1305/ndjfl/1093891789.

[18] A. Indrzejczak, Two Is Enough - Bisequent Calculus for S5, [in:] A. Herzig, A. Popescu (eds.), Frontiers of Combining Systems, Springer International Publishing, Cham (2019), pp. 277-294, DOI: https://doi.org/10.1007/ 978-3-030-29007-8_16.

[19] A. Indrzejczak, Sequents and Trees. An Introduction to the Theory and Applications of Propositional Sequent Calculi, Studies in Universal Logic, Birkhäuser, Basel (2021), DOI: https://doi.org/10.1007/9783-030-57145-0.

[20] K. Ishii, R. Kashima, K. Kikuchi, Sequent Calculi for Visser's Propositional Logics, Notre Dame Journal of Formal Logic, vol. 42(1) (2001), pp. 122, DOI: https://doi.org/10.1305/ndjfl/1054301352.

[21] G. Kalmbach, Orthomodular Lattices, Academic Press, New York (1983).

[22] T. Kawano, Advanced Kripke Frame for Quantum Logic, [in:] L. S. Moss, R. de Queiroz, M. Martinez (eds.), Logic, Language, Information, and Computation, Springer Berlin Heidelberg, Berlin, Heidelberg (2018), pp. 237-249, DOI: https://doi.org/10.1007/978-3-662-57669-4_14. 
[23] T. Kawano, Labeled Sequent Calculus for Orthologic, Bulletin of the Section of Logic, vol. 47(4) (2018), pp. 217-232, DOI: https://doi.org/10. 18778/0138-0680.47.4.01.

[24] S. Negri, Proof Theory for Modal Logic, Philosophy Compass, vol. 6(8) (2011), pp. 523-538, DOI: https://doi.org/10.1111/j.17479991.2011.00418.x.

[25] H. Nishimura, Sequential method in quantum logic, Journal of Symbolic Logic, vol. 45(2) (1980), pp. 339-352, DOI: https://doi.org/10.2307/ 2273194.

[26] H. Nishimura, Proof Theory for Minimal Quantum Logic I, International Journal of Theoretical Physics, vol. 33(1) (1994), pp. 103-113, DOI: https://doi.org/10.1007/BF00671616.

[27] H. Nishimura, Proof Theory for Minimal Quantum Logic II, International Journal of Theoretical Physics, vol. 33(7) (1994), pp. 1427-1443, DOI: https://doi.org/10.1007/BF00670687.

[28] H. Ozawa, H. Odera, S. Chitani, Formal System QL for Quantum Logic, The Japan Association for Logical Philosophy, Logical Philosophy Research, vol. 3 (2003).

[29] M. Ozawa, Operational Meanings of Orders of Observables Defined through Quantum Set Theories with Different Conditionals, Electronic Proceedings in Theoretical Computer Science, vol. 236 (2017), pp. 127-144, DOI: https://doi.org/10.4204/eptcs.236.9.

[30] F. Poggiolesi, Gentzen Calculi for Modal Propositional Logic, vol. 32 of Trends in Logic, Springer, Dordrecht (2011).

[31] A. Visser, A Propositional Logic with Explicit Fixed Points, Studia Logica, vol. 40(2) (1981), pp. 155-175, DOI: https://doi.org/10.1007/BF01874706.

\section{Tomoaki Kawano}

Tokyo Institute of Technology

School of Computing

Department of Mathematical and Computing Science

2-12-1 Okayama, Meguro-ku

Tokyo, Japan

e-mail: kawano.t.af@m.titech.ac.jp 